\title{
ESTUDIO DEL MATERIAL REFRACTARIO NATURAL DE TARATA - TACNA
}

\section{STUDY OF NATURAL REFRACTORY MATERIAL FROM TARATA - TACNA}

\author{
${ }^{1}$ Carlos Huisa Ccori; ${ }^{2}$ Gualberto Tejada Bedoya; ${ }^{3}$ Zenón Sarmiento Mejia
}

\begin{abstract}
RESUMEN
Mucho de los refractarios naturales y especiales cubren solamente mercados muy limitados, pero al mismo tiempo los usuarios especializados están continuamente buscando nuevos materiales sobresalientes para ayudarles a resolver muchos problemas de diseño en las tecnologías nuclear, aviaciön, coheteria, hornos especiales etc. Los materiales buscados tienen que sustituir y actuar mejor que los metales ya disponibles a las temperaturas más elevadas que pueda alcanzar. En este sentido se ha examinado todo el campo de posibilidades; no solamente óxidos, sino también nitruros, carburos, bromuros e incluso sulfuros. Probablemente pocos de ellos encontraron aplicación en la tecnología de hornos. En el presente trabajo según el análisis fisicoquimico de la roca Piroclástica (cristobalita en estado Natural) demuestra que es una roca refractaria con $1,33 \mathrm{gr} / \mathrm{cm}^{3}$ de densidad, $3,39 \%$ de Humedad, $35,73 \%$ de porosidad, $13,01 \mathrm{KN} / \mathrm{m}^{3}$ de peso especifico, con resistencia a compresión simple 5,0
Mpay difracción de rayos $X$ de $22 \%$ de cristobalita.
\end{abstract}

Palabras Claves: Refractario, roca Piroclástica, Cristobalita

\section{ABSTRACT}

Much of the natural and special refractory material cover only very limited markets, but also specialized users are constantly looking for new outstanding materials to help them solve many design problems in nuclear technology, aviation, rocketry, ovens etc. The wanted materials have to replace and do better than already available metals and at higher temperatures that can reach. In this sense all the range of possibilities has been examined not only oxides, but also nitrides, carbides, sulfides and even bromides. Probably few of them found application in furnace technology. In this paper according to the Physicochemical analysis of pyroclastic rock (cristobalite in natural state) shows that it is a refractory rock with $1.33 \mathrm{~g} / \mathrm{cm}^{3}$ density, moisture $3.39 \%, 35.73 \%$ porosity, $13.01 \mathrm{kN} / \mathrm{m}^{3}$ of specific weight, compressive strength 5.0 MPa.XRD and $22 \%$ cristobalite.

Key Words: Refractory, pyroclastic rock, cristobalite

\section{INTRODUCCIÓN}

La sílice se presenta en la naturaleza como seis minerales distintos: el cuarzo, la calcedonia, el ópalo, la tridimita, la cristobalita y la lechatelierita (vidrio de sílice). De estos, el cuarzo es muy común; la tridimita y la cristobalita se encuentran ampliamente distribuidas en las rocas volcánicas; la calcedonia (criptocristalina) y el ópalo (amorfo) son de ocurrencia extensa; la lechatelierita es muy rara. (Mason, 1958).

El cuarzo es la forma estable de la sílice a la presión atmosférica, a temperaturas hasta de $867{ }^{\circ} \mathrm{C}$, la tridimita entre 867 y $1470{ }^{\circ} \mathrm{C}$ y la cristobalita desde $1470^{\circ} \mathrm{C}$ hasta su punto de fusión a $1713^{\circ} \mathrm{C}$. La sílice liquida es la fase estable. El presente trabajo de investigación tiene como objetivo demostrar que la roca Piroclástica (cristobalita) de Tarata Susapaya es una roca refractaria.

\subsection{Objetivos Principal}

Realizar un análisis físico - químico de las rocas volcánicas que se encuentran en la Zona de Tarata - Tacna para ser utilizado como material refractario.

\subsection{Objetivos Secundarios}

Realizar un estudio exploratorio de la zona de Tarata para ubicar el yacimiento de Rocas Volcánicas.

Realizar un análisis de las características físicas con ensayos de Laboratorio.

Realizar un análisis Dx-R para determinar la refracción del material en estudio.

\subsection{Justificación del Problema}

Según J.D. Gilchrist (1967) no existe una definición general del refractario. Esencialmente son materiales de punto de fusión elevado; sin embargo, es un aspecto relativo, y el punto de fusión no es el único criterio de utilidad. 
La mayor parte de los refractarios son materias cerámicas, fabricadas con óxidos de elevado punto de fusión, particularmente $\mathrm{SiO} 2, \mathrm{Al} 2 \mathrm{O} 3, y \mathrm{Mg} \mathrm{O}$. No obstante, el carbón es actualmente un refractario importante, y los carburos, nitruros y bromuros se están también desarrollando para el trabajo a altas temperaturas.

En ese sentido definimos que los refractarios son materiales bastante utilizados en la industria metal mecánica (fundición) como en los hornos domésticos, etc. Por tal motivo es necesario investigar si las rocas volcánicas descubiertos en la zona de Tarata pudiera ser utilizado en forma natural como refractario.

\section{MATERIALES Y MÉTODOS}

\subsection{Propiedades y tipos de ensayos}

Las propiedades más importantes requeridos por el usuario son:

$1^{\circ}$ Rigidez y permanencia del tamaño, forma y resistencia mecánica a la temperatura de operación que será elevado.

$2^{\circ}$ Capacidad para resistir un choque térmico, tal como ocurre en la calefacción y enfriamiento de hornos.

$3^{\circ}$ Se exige frecuentemente resistencia al ataque químico por cualquier clase de gas, escoria o metal.

Como no existe ningún material capaz de resistir cualquier condición posible, se debe elegir para satisfacer las exigencias de la obra en cuestión. Normalmente es preciso aceptar una situación de compromiso.

Normalmente se efectúan los ensayos siguientes. Considerando las especificaciones de BS (British Standards Specifications) para algunos marcados con asteriscos y se pueden encontrar detalles en BSS 1902 (1952) o en un apéndice de "Steel Plant Refractories". Una serie de ensayos análogos están especificados por la ASTM. (American Society for Testing Materials).

- Examen Visual

- Exactitud de dimensiones

- Dilatación remanente

- Dilatación térmica reversible

- Refractariedad

- Resistencia mecánica en frío

- Resistencia mecánica en caliente

- Resistencia a la escoria

- Porosidad

- Densidad real

- Permeabilidad

- Análisis Químico

- Petrografía

\subsection{Diseño de la investigación}

Población.-Zona de Tarata-Tacna.

Muestra.-Calicatas en forma sistemática

Análisis Físico - Químico en los Laboratorios de Mecánica de rocas de la FAIN

\subsection{Principales trabajos ejecutados en el campo y laboratorio.}

Visita de reconocimiento, Estudios Topográficos, Investigación Geotécnico detallado, Sondeos y muestreo, Ensayos de campo, Ensayos de Laboratorio

\section{RESULTADOS}

\subsection{Propiedades físicas de la roca piroclástica (Cristobalita)}

Tabla $N^{\circ} 01$. Densidad.

\begin{tabular}{lccccc}
\hline Muestra & $\begin{array}{c}\text { Peso } \\
\mathrm{gr}\end{array}$ & $\begin{array}{c}\text { Diámetro } \\
\mathrm{cm}\end{array}$ & $\begin{array}{c}\text { Altura } \\
\mathrm{cm}\end{array}$ & $\begin{array}{c}\text { Volumen } \\
\mathrm{cm}^{3}\end{array}$ & $\begin{array}{c}\text { Densidad } \\
\mathrm{gr} / \mathrm{cm}^{3}\end{array}$ \\
\hline Roca Piroclástica & 117,6 & 3,78 & 7,90 & 88,65 & 1,33 \\
\hline
\end{tabular}

Tabla N02. Humedad

\begin{tabular}{ccccc} 
Muestra & $\begin{array}{c}\text { Peso húmedo } \\
\mathrm{W}_{\mathrm{b}} \\
\mathrm{gr}\end{array}$ & $\begin{array}{c}\mathrm{W}, \\
\mathrm{gr}\end{array}$ & $\begin{array}{c}\mathrm{W}_{\mathrm{h}}= \\
\mathrm{W}\end{array}$ & $\begin{array}{c}\text { Humedad } \\
\mathrm{W}=\left(\mathrm{W}_{\mathrm{w}} / \mathrm{W}\right) \mathrm{x} \\
100 \%\end{array}$ \\
\hline Roca Piroclástica & 204,1 & 197,4 & 6,7 & 3,39 \\
\hline
\end{tabular}

Tabla No03. Peso específico

\begin{tabular}{ccccccc}
\hline Muestra & $\begin{array}{c}\text { Peso Diámetro } \\
\mathrm{gr}\end{array}$ & $\begin{array}{c}\text { Altura } \\
\mathrm{cm}\end{array}$ & $\begin{array}{c}\text { Volumer } \\
\mathrm{cm}^{3}\end{array}$ & $\begin{array}{c}\text { Densidad } \\
\mathrm{gr} / \mathrm{cm}^{3}\end{array}$ & $\begin{array}{c}\text { Peso } \\
\text { Especifico } \\
\mathrm{KN} / \mathrm{m}^{3}\end{array}$ \\
\hline $\begin{array}{c}\text { Roca } \\
\text { Piroclástica }\end{array}$ & 117,6 & 3,78 & 7,9 & 88,65 & 1,33 & 13,01 \\
\hline
\end{tabular}

Tabla $N^{\circ} 04$. Volumen de poros, porosidad, $\%$

\begin{tabular}{|c|c|}
\hline $\mathrm{N}^{\circ}$ Características & $\begin{array}{c}\text { Muestra: } \\
\text { Roca Piroclástica } \\
\end{array}$ \\
\hline 1 Peso saturado $\mathrm{M}_{\mathrm{su}}(\mathrm{gr})$ & 115,4 \\
\hline 2 Peso seco $M_{r}$ (gr) & 89,8 \\
\hline 3 Densidad del agua $\mathrm{P}\left(\mathrm{gr} / \mathrm{cm}^{3}\right)$ & 1,0 \\
\hline 4 Volumen del cilindro $V\left(\mathrm{~cm}^{3}\right)$ & 71,64 \\
\hline 5 Volumen de Poros $V_{v}=\left(M_{s-1}-M_{1}\right) / P$ & 25,6 \\
\hline $\mathrm{n}=\left(\mathrm{V}_{*} / \mathrm{V}\right) \times 100$ & 35,73 \\
\hline $7 \%$ de Absorción $\mathrm{W}=(\mathrm{Mat}-\mathrm{Ms}) / \mathrm{Ms}) \times 100(\%)$ & $28,51 \%$ \\
\hline
\end{tabular}

\subsection{Propiedades mecánicas de la roca piroclástica}

Tabla $\mathrm{N}^{\circ} 05$. Ensayo de tracción (método brasileiro)

\begin{tabular}{|c|c|c|c|c|c|c|}
\hline Muestra & $\begin{array}{c}\text { Fecha de } \\
\text { rotura }\end{array}$ & $\begin{array}{c}\text { Diámetro } \\
\mathrm{cm}\end{array}$ & $\begin{array}{c}\text { Long. } \\
\mathrm{cm}\end{array}$ & $\begin{array}{c}\text { Fuerza Aplic } \\
\mathrm{Kg}\end{array}$ & $\begin{array}{c}\sigma_{\mathrm{c}} \\
\mathrm{Kg} / \mathrm{cm}^{2}\end{array}$ & $\begin{array}{c}\delta_{c} \\
\mathrm{Mpa}\end{array}$ \\
\hline Roca & $10 / 05 / 13$ & 3,79 & 1,80 & 15 & 1,40 & 0,14 \\
\hline
\end{tabular}

Tabla N06. Compresión simple

\begin{tabular}{ccccccr}
\hline Muestra & $\begin{array}{c}\text { Fecha de } \\
\text { rotura }\end{array}$ & $\begin{array}{c}\text { Diámetro } \\
\mathrm{cm}\end{array}$ & $\begin{array}{c}\text { Long } \\
\mathrm{cm}\end{array}$ & $\begin{array}{c}\text { Fuerza Aplic. } \\
\mathrm{Kg}\end{array}$ & $\begin{array}{c}\sigma_{c} \\
\mathrm{Kg} / \mathrm{cm}^{2}\end{array}$ & $\begin{array}{c}\sigma_{c} \\
\mathrm{Mpa}\end{array}$ \\
\hline $\begin{array}{c}\text { Roca } \\
\text { Piroclástica }\end{array}$ & $10 / 05 / 13$ & 3,78 & 7,90 & 571,5 & 50,93 & 5,0 \\
\hline
\end{tabular}

Ver Figura $\mathrm{N}^{\circ} 01$ Ángulo de Fricción Interna y cohesión de la Roca.

\subsection{Análisis por difracción de rayos $\mathrm{X}$}

Tabla $N^{\circ} 07$. Análisis por difracción de rayos $\mathrm{X}$

\begin{tabular}{ccc} 
Nombre del mineral & Formula General & $\begin{array}{c}\text { Resultado } \\
\text { Aproximado (\%) }\end{array}$ \\
\hline Plagioclasa (Andesina) & $(\mathrm{Ca}, \mathrm{Na})(\mathrm{Nl}, \mathrm{Si}) 4 \mathrm{O} 8$ & 36 \\
Feldespato (Ortoclasa) & $\mathrm{KAlSi} 3 \mathrm{O} 8$ & 29 \\
Cristobalita & $\mathrm{SiO} 2$ & 22 \\
Cuarzo & $\mathrm{SiO} 2$ & 7 \\
Zeolita (Erionita) & $\mathrm{Na} 2, \mathrm{~K} 2, \mathrm{Ca}) 2[\mathrm{Al} 4 \mathrm{Si} 14 \mathrm{O} 36]^{* 15}(\mathrm{H} 2 \mathrm{O})$ & $<\mathrm{L} . \mathrm{D}$. \\
Esmectita & $-\mathrm{L} . \mathrm{D}$. \\
Mica (Biotita) & $\mathrm{K}(\mathrm{Mg}, \mathrm{Fc}) 3[\mathrm{AlSi} 3 \mathrm{O} 10(\mathrm{OH}, \mathrm{F}) 2]$ & $<\mathrm{L} . \mathrm{D}$. \\
Hematita & $\mathrm{Fe} 2 \mathrm{O} 3$ & $<\mathrm{L} . \mathrm{D}$.
\end{tabular}

Rexp: 3.14 Rwp: 7.58 GOF: 2.42 Valor de Aproximación $=1 \%$ 
3.4. Análisis de dilatación refractaria de la roca piroclástica (Cristobalita)

Ver Figura $\mathrm{N}^{\circ} 02$.

Tabla Nº8. Análisis de dilatación refractaria de la roca piroclástica

\begin{tabular}{llllllll}
\hline Ensayo & 1 & 2 & 3 & 4 & 5 & 6 & 7 \\
\hline $\mathrm{T}^{\circ} \mathrm{C}^{\circ}$ & 0 & 200 & 400 & 600 & 800 & 1200 & 1400 \\
DILAT. $\mathrm{mm}$ & 0,0 & 0,50 & 1,1 & 1,2 & 1,3 & 1,3 & 1,3 \\
\hline
\end{tabular}

\section{DISCUSIÓN}

Sosman (1927) afirma que existen quince modificaciones de silice, las formas mas importantes se

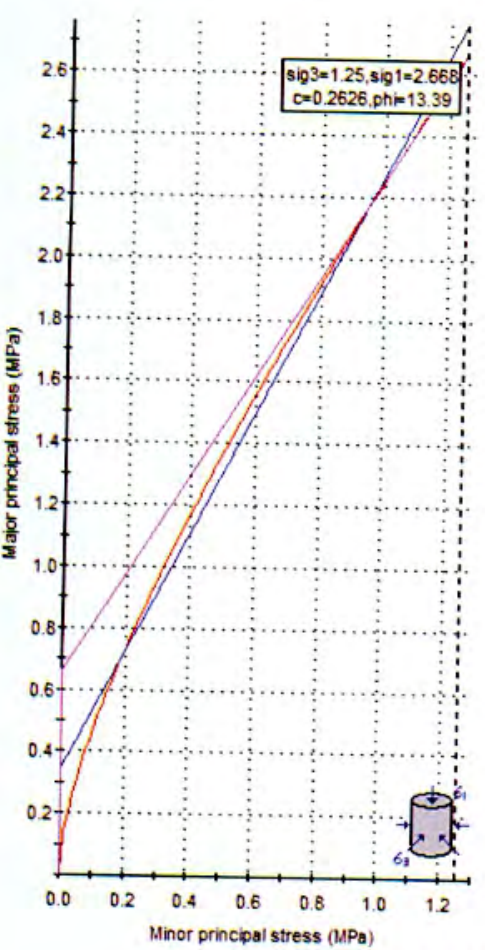

han dispuesto en la Figura $\mathrm{N}^{\circ} 01$, mostrando sus intervalos de estabilidad y los cambios de volumen que ocurren en las transformaciones. La clasificación de Sosman muestra la conversión de cuarzo y tridimita mas bien a vidrio que a cristobalita a 1450 y $1680 \mathrm{C}^{\circ}$ respectivamente. El estado líquido, bajo enfriamiento, forma vidrio o silice vitrea, que es, por supuesto, una forma metaestable, y desvitrificación a cristobalita.

Cuando se enfría la cristobalita, por debajo de 1470 ${ }^{\circ} \mathrm{C}$, puede volver a tridimita, pero este paso es lento $y$ normalmente persiste como cristobalita metaestable hasta la temperatura ambiente, si bien sufre una transformación compleja a $275-200^{\circ} \mathrm{C}$, en una variedad de baja temperatura.

Según la Figura $\mathrm{N}^{\circ} 04$ la desvitrificación del vidrio se produce siempre a cristobalita incluso a temperaturas
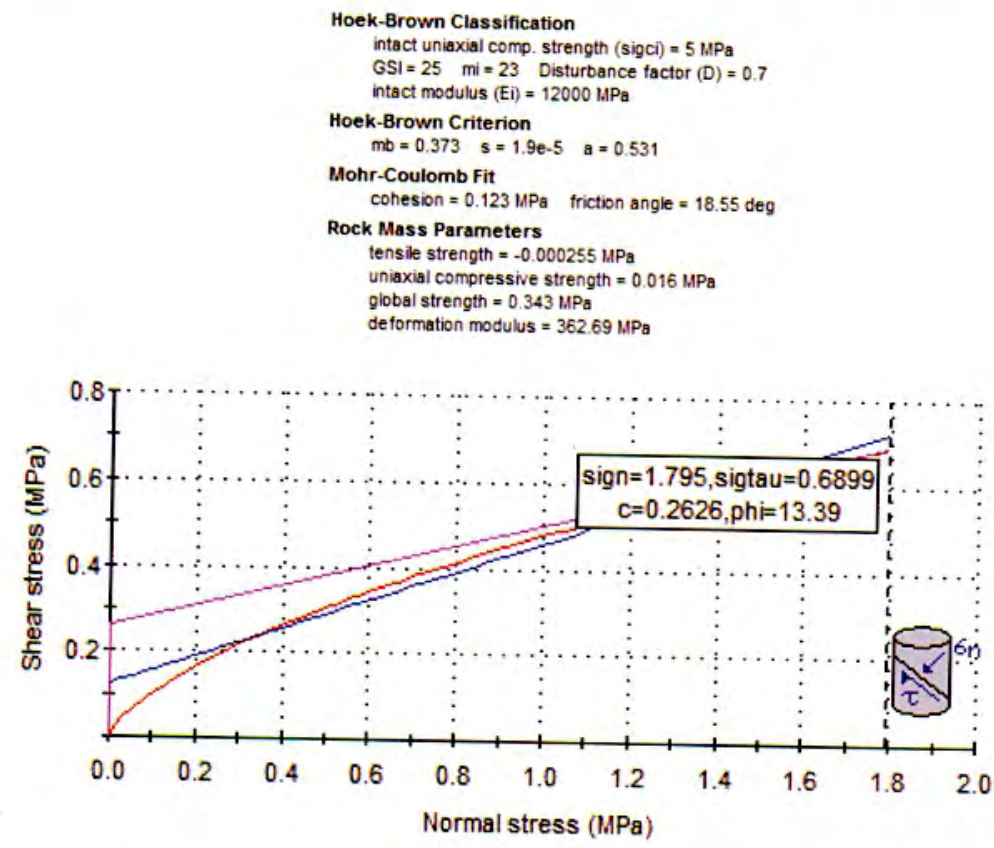

Figura $\mathrm{N}^{\circ} 01$. Ángulo de fricción y cohesión de la roca piroclástica Fuente: Elaboración propia

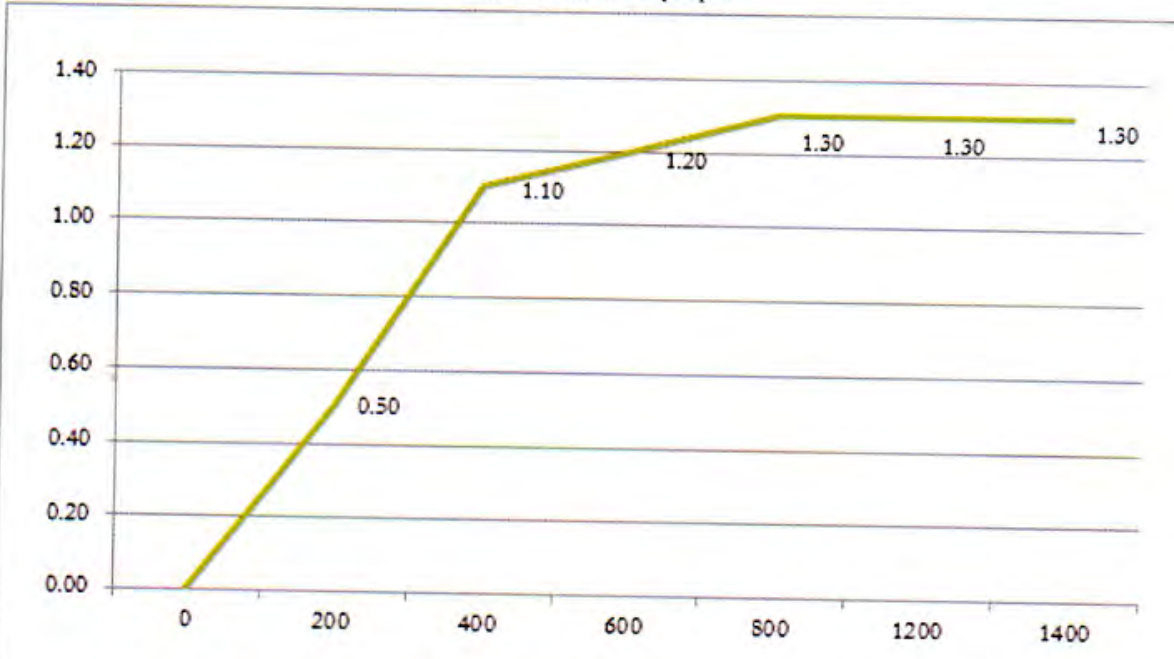

Figura $N^{\circ}$ 02. Análisis de Dilatación de la Roca Piroclástica 


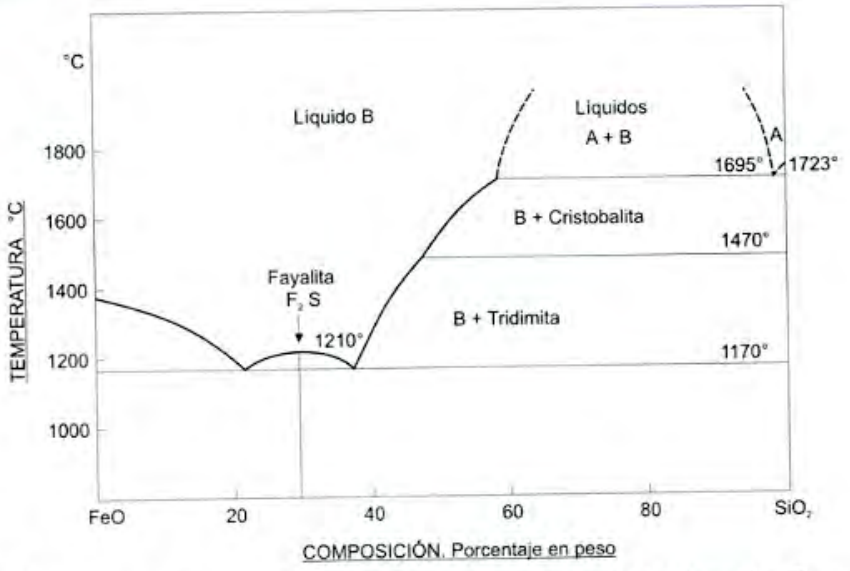

Figura $\mathrm{N}^{\circ}$ 03. Diagrama de equilibrio del sistema $\mathrm{FeO}-\mathrm{SiO}_{2}$ (según Bowen y Schairer)

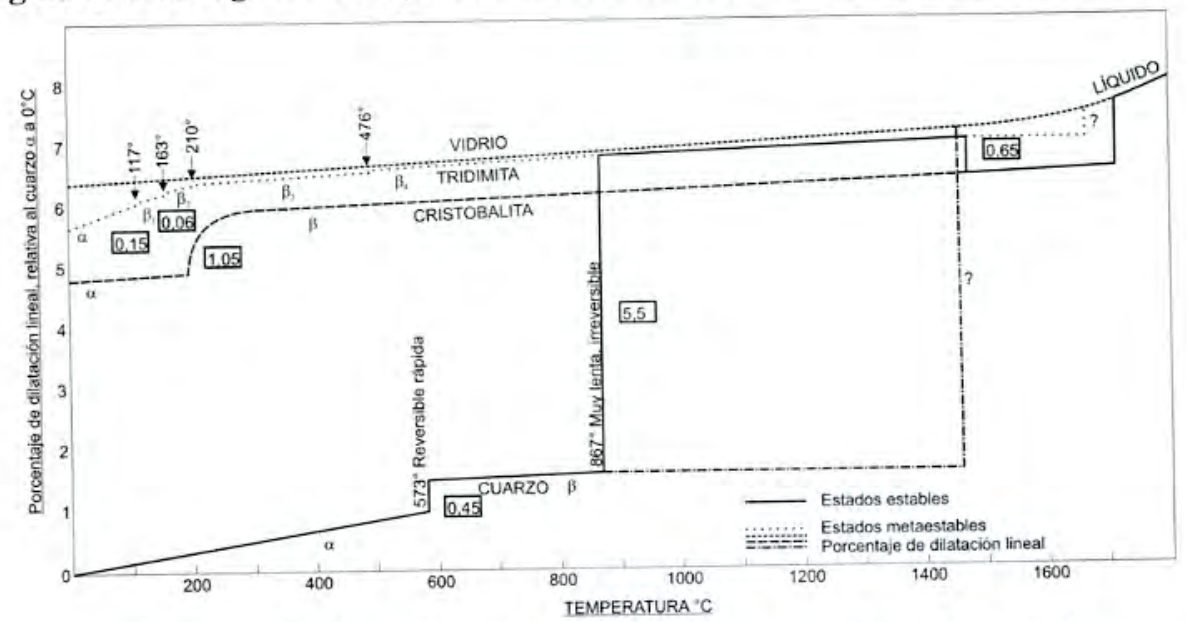

Figura $\mathrm{N}^{\circ}$ 04. Alotropía de la sílice y cambios de volumen asociados

inferiores a $1470^{\circ} \mathrm{C}$, a las cuales la cristobalita es metaestable. De forma similar, la cristobalita se encuentra en las briquetas refractarias mejor que la tridimita, incluso después de una cocción por debajo de $1470^{\circ} \mathrm{C}$. Ésta descripción de Sosman reafirma a la roca Piroclástica de Tarata que según el análisis de difracción de Rayos X determina que tiene $22 \%$ de Cristobalita.

Según Bowen y Schairer (1958) presenta la Figura $\mathrm{N}^{\circ} 03$ en donde se puede apreciar que la tridimita trabaja a una temperatura aproximado de $1170^{\circ} \mathrm{C}$ a $1470^{\circ} \mathrm{C}$, mientras que la cristobalita según el contenido de sílice trabaja desde 1470 a $1695^{\circ} \mathrm{C}$. La roca Piroclástica de Tarata (Zona de Investigación) estaría cumpliendo este rango.

\section{V.CONCLUSIONES}

Según el estudio Geológico Exploratorio de la zona determina una reserva superior a 24 Millones de Toneladas. Y el método de Explotación de éste producto será por cielo abierto (cantera)

La roca Piroclástica (roca volcánica de Tarata Susapaya) llamada cristobalita es una variedad de Silice que tiene propiedades de fundición superior a $1470^{\circ} \mathrm{C}$ y con una dilatación refractaria de $1,30 \mathrm{~mm}$.

Por su bajo nivel de densidad $1,33 \mathrm{gr} / \mathrm{cm}^{3}$ se puede utilizar en la fabricación de briquetas livianas para la construcción de la bóveda de los Hornos.

Según el análisis Fisicoquímico de la Roca Piroclástica (cristobalita en estado Natural) demuestra que es una roca refractaria con: $1,33 \mathrm{gr} / \mathrm{cm}^{3}$ de densidad, $3,39 \%$ de Humedad, $35,73 \%$ de porosidad, $13,01 \mathrm{KN} / \mathrm{m}^{3}$ de peso específico, con resistencia a compresión simple 5,0 Mpa y difracción de rayos $\mathrm{X}$ de $22 \%$ de cristobalita.

\section{REFERENCIAS BIBLIOGRÁFICAS}

R. B. Sosman (1927). "The properties of Silice" American Chemical Society Monograph. EEUU.

J.D. Gilchrist (1967). "Combustibles y refractarios".

Editorial Alhamora S.A. Madrid España.

B.S.S. Norton (1902) Cerámica Fina, Tecnología y

Aplicaciones. Editorial Omega. España.

J.K. Mason (1958) Steel Plant Refractories. Editorial

American Society for Testing Materials. Canada-EEUU.

Bowen y Schairer (1958) Refractories. Editorial American

Society for Testing Materials. Canada-EEUU.

\section{Correspondencia:}

Carlos Huisa Ccori: chuisa_54@hotmail.com
Fecha de Recepción: 09/05/2014

Fecha de Aceptación: 18/06/2014 